$\xi=-$ 圆

\title{
Load Balancing Metric Based Routing Protocol for Low Power and Lossy Networks (lbRPL)
}

\author{
A. Sebastian ${ }^{1}$, S. Sivagurunathan ${ }^{2 *}$ \\ ${ }^{1,2 *}$ Department of Computer Science and Applications, Gandhigram Rural Institute \\ *Corresponding author E-mail: s.sivagurunathan@ ruraluniv.ac.in
}

\begin{abstract}
IETF ROLL working Group standardized the IPv6 Routing protocol (RPL) for applications over low-power and lossy networks (LLNs). RPL constructs a Destination Oriented Direction Acyclic Graph (DODAG) to organize network topology. RPL shows fast network setup and good scalability. However, it may suffer from load imbalance due to diverse network traffic and heavy load on preferred or forwarding parents. To optimize the load balancing of routes in RPL, this paper proposes load balancing metric based routing protocol called lbRPL. We introduce a new routing metric for RPL called load balancing index (LBI), which exploits load balancing characteristics of RPL nodes to select more load balanced parents and routes. LBI includes ETX, Parent count (Pc) and Remaining Parent Energy (Pe) metrics to make routing decisions. Simulation results show that lbRPL improves network performance, stability and improved network life time to RPL.
\end{abstract}

Keywords: Internet of Things, RPL, Load Balancing Index, Load Balancing Characteristics

\section{Introduction}

Low power and lossy networks (LLNs) are providing innovative applications such as smart building [1], habitat monitoring, smart city [2] and industrial automation. Since LLNs are composed of large number of resource constrained nodes, routing is challenging task. Resource constraints include memory, processing power, power, low data rate and lossy environment. Lossy wireless communication environment makes RPL network topology unstable, load imbalanced which result in imbalanced routing and poor performance.

In 2014, the IETF ROLL working group standardized routing requirements for four application scenario: home automation (RFC 5826), Industrial control (RFC 5673), urban environment (RFC 5548) and building automation (RFC 5867) [3]. RPL is a distance vector routing protocol, in which nodes construct a Destination Oriented Directed Acyclic Graph (DODAG) by exchanging distance vectors from the root. On the one hand, performance evaluation of RPL by researchers suggest fast network setup [4] and good scalability but RPL network suffers from load imbalance as shown by [5] and [6]. Load imbalance introduces frequent parent change which have adverse impact on the performance of RPL network. RPL uses objective function (OF) to guide nodes to select preferred parents and construct DODAG topology. Several routing metrics such as hop count and ETX are specified [7]. However, load balancing objective function are a few.

Load Imbalance of RPL network lead to problems such as bottle neck, energy hole, thundering herd, early node death and poor network performance. These problems can affect the RPL network badly if the affected nodes are one hop to the sink or root. Therefore, efficient load balancing mechanisms are needed to be devised to thwart these issues.

In this article, we propose a load balancing metric based RPL (lbRPL) to select load balanced routes in LLNs. To obtain load bal- ance in RPL, we introduce a new routing metric called load balancing index (LBI). Based on LBI, lbRPL selects load balanced nodes as their parents to form more load balanced routes. lbRPL calculates the parent count before choosing the preferred parent. Hence, LBI, ETX and Node remaining energy are used as a routing metric to build reliable routes.

The rest of this paper is organized as follows. Section II presents related work. Section III describes RPL over view and load balancing characteristics of RPL. Section IV explains LBI design and properties. Section $V$ gives details on network simulation set up and performance evaluation. Section VI concludes the work with future load balancing challenges.

\section{Related Work}

In multi-hop wireless networks, choosing proper routing metric to discover load balanced routes pose challenges to network layer routing protocols. In RPL, the commonly used network layer metrics are hop count and ETX. However, minimum number of hops or low ETX value does not necessarily provide load balancing. RPL also lacks load balancing performance metrics. Therefore, we need to design composite metrics keeping in mind node and link characteristics that support load balancing. Many authors have suggested solutions to load balance RPL network. Their area of focus is improving stability of the network, extended network life time, avoid bottleneck, distribute load and improved network performance. We list here, important works related to load balancing in RPL. In [8], the authors suggested queue utilization (QU-RPL). QU-RPL is designed for each node to select its parent node considering the queue utilization of its neighbour nodes as well as their hop distances to an LLN border router (LBR). QU-RPL is effective in lowering queue losses and increasing the packet delivery ratio compared to the standard RPL. 
In [9] the authors propose, Minimum Degree RPL (MD-RPL) which builds a minimum degree spanning tree to enable load balancing in RPL. MD-RPL modifies the original tree formed by RPL to decrease its degree.

In [10], the authors proposed a load balanced routing protocol based on the RPL protocol (LB-RPL) to achieve balanced workload distribution in the network. LB-RPL detects workload imbalance in a distributed and non-intrusive fashion. It also optimizes the data forwarding path by jointly considering both workload distribution and link-layer communication qualities.

In [11], the authors designed an energy-balancing routing protocol that maximizes the lifetime of the most constraint nodes. They proposed the Expected Lifetime metric, denoting the residual time of a node (time until the node will run out of energy). They also designed mechanism to detect energy-bottleneck nodes and to spread the traffic load uniformly among them.

In [12] the authors propose three multipath schemes based on RPL: Energy Load Balancing (ELB), Fast Local Repair (FLR) and their combination (ELB-FLR).

In [13] the authors address the imbalance of traffic load among gateways. The load balancing between gateways is suggested to reduce the traffic congestion thereby enlarging the network capacity. They proposed dynamic and distributed load balancing scheme to achieve a global load fairness motivated by water flow behaviour named Multi-Gateway Load Balancing Scheme for Equilibrium (MLEq).

In [14], the authors design energy balancing routing protocol that maximizes the life time of the most constrained nodes. They proposed expected life time metric that suggests residual time of a node and hence detect energy-bottleneck nodes and to spread the traffic load uniformly among all nodes.

In [14], the authors suggested neighbourhood metric that would suggest quality of neighbouring nodes along with current forwarding route. Current forwarding is compared with neighbouring nodes for ETX. Hence improved load balancing is obtained.

In [15], the authors suggested Heuristic Load distribution algorithm (HeLD) which achieves a balanced traffic load and improved life time when throughput is high. This is based on braided multipath RPL extension technique.

In [16], the authors proposed a new objective function based on MHROF. The new OF uses both the traffic profile of the nodes and the ETX of the links in order to solve imbalance problem. The proposal is called A LoAd BAlancing Model for RPL (ALABAMO). It improves the network life time.

In [17], the authors proposed multi sink for improved network life time. Multi sink technique allows more node to participate in the network which reduces the number of hop thus resulting in improved life time. In [18], the authors suggested Bandwidth Allocation based RPL Load Balancing (BA-LBRPL). They considered network resource like bandwidth for parent selection.

In this article, we introduce a new routing metric called Load Balancing Index (LBI) for RPL protocol to discover load balanced routes in LLNs. Nodes and data sinks form a large scale wireless mesh network in which nodes typically send their data packet to data sinks in a multi-hop manner. Based on LBI, a leaf node first selects the parent with lowest parent count, lowest ETX and Low high on remaining energy. Hence LBI is used to calculate rank and construct DODAG in RPL.

\section{Load Balancing Characteristics in RPL}

In this section, we give brief overview of Routing Protocol for Low Power and Lossy networks (RPL) and explore load balancing characteristics.

\subsection{RPL Overview}

In general, an RPL-based network consists of three types of nodes: root node, connecting to another network as a gateway or border router (node R); router (nodes A, B, C, D, E, F, G), forwarding topology information and data packets to their neighbours; leaf node (nodes H, I, J and K), only joining a DODAG as an end member. This is explained in figure 1 .

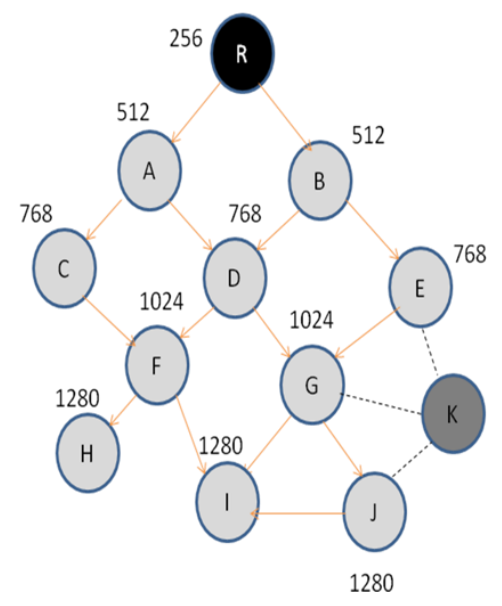

Figure 1: DODAG construction in RPL

The construction of a DODAG starts at the root node (R), through the routers, down to the leaf nodes. The root node broadcasts to its sub nodes the DODAG information Object (DIO) messages that contain RANK information (like $256,512, \ldots 1280$ ). Once receiving DIO messages, a leaf node ( node $\mathrm{k}$ ) can decide whether to join preferred parents $\mathrm{E}, \mathrm{G}$ and $\mathrm{J}$. The leaf node $\mathrm{k}$, calculates rank according to the equations (1) and (2) [RFC 6719]. [11]

$\operatorname{Rank}(\mathrm{N})=\operatorname{Rank}(\mathrm{PN})+\operatorname{RankIncrease}$

RankIncrease $=$ Step $*$ MinHopRankIncrease

Where Step represents a scalar value and MinHopRankIncrease represents the minimum RPL parameter. If the node $\mathrm{k}$ decides to join, then it adds the DIO sender to the candidate parent list. Next, the preferred parent, i.e. the next hop to the root, will be chosen based on the rank from this list to receive all traffic from the leaf node. Then, it computes its own rank with a monotonical increase according to the selected OF, in our case LBI. After that, the node propagates its own DIO with all updated information to all its neighbors including the preferred parent. This process is repeated till a path from leaf node to the root is constructed in the form of Destination Oriented Directed Acyclic Graph (DODAG).

\subsection{Load Balancing characteristics in RPL}

Load balancing characteristics in RPL can be explained by figure 2 . The network topology consists of a root node (BR). Nodes K and L are routers or preferred parent nodes to A, B, C, D and E. The small circles are leaf nodes waiting to join the DODAG by receiving RANK properties from the parent nodes K and L. Due to the lack of balance algorithm, many leaf nodes may select the same parent node and leave other with a few nodes. In this case, parent $\mathrm{K}$ has 3 nodes in its range and $\mathrm{L}$ has 2 nodes in its communication range. It is observed that many leaf nodes get attached to Parent $\mathrm{K}$ which already has more child nodes. As a result, parent $\mathrm{L}$ will have few child nodes. At the next interval, increment in RANK of parent nodes will triggers all child nodes to re-select and switch their parent node. This way frequent switching of parent nodes greatly decrements the network efficiency, unstable topology and deplete constrained resources. Here we suggest load balancing characteristics before we proceed to design load balancing (LBI) metric based routing for RPL. 


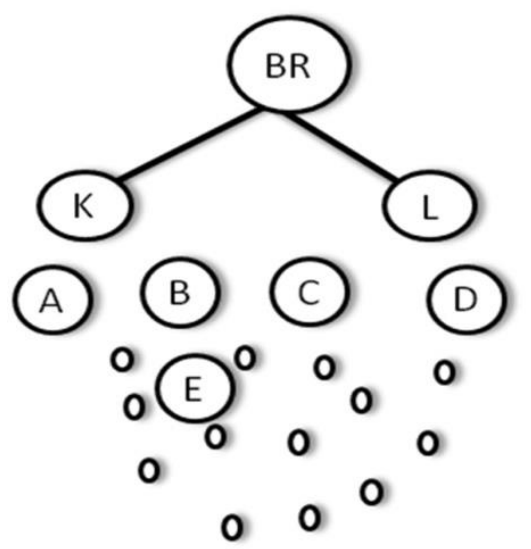

Figure 2: Load Imbalance in RPL

\subsubsection{Stability}

RPL based networks are scalable networks with large number of nodes. For efficient network performance, network stability is an important parameter. Since RPL uses trickle timer to minimize control traffic overhead, network instability will initiate local /global repair mechanisms often. Frequent reboot of trickle timer will result in unnecessary use of network resources like memory and power. This scenario will generate more number of control traffic. Therefore, when the RPL network is stable it gives efficient performance for all IoT scenario.

\subsubsection{Parent Switching}

Instability in RPL is the result of load imbalance among various link routes. RPL uses RANK property to select parent nodes. When new nodes receive DIO from preferred parents it would update its rank with low Rank of the DIO received from preferred parent. For example, in figure 1 , new node $\mathrm{K}$ has three DIO message from parent nodes $\mathrm{E}, \mathrm{G}$ and $\mathrm{J}$ and it would update its rank with DIO from parent node $\mathrm{E}$ as it has the lowest rank. Hence, many nodes will join the lower rank parent. This will increase the rank value of the parent. In the next interval if some other parent has low rank, the nodes from the previous parent will leave that parent and join the new parent. This way at every network setup the leaf nodes keep switching their parent. This can hamper the efficiency of RPL performance.

\subsubsection{Improved Life Time}

Load Balancing problems in RPL such as energy hole, bottleneck, thundering herd, hotspot, etc. directly affect the node and link resources. Early node death, energy depletion, high occupation of bandwidth, buffer occupancy are results of load imbalance. Hence, load balancing algorithms need to address these problems so that RPL network has improved node and network life time.

\subsubsection{Balanced Load Distribution}

Many of the problems related to load imbalance in RPL puts uneven load among the nodes, routers in the network. Since RPL is a mesh and multi-hop network, more load on individual nodes affect not only that node but also the entire network. Therefore, there is a great need to identify the individual node and link that has more load. Designing routing algorithms that would distribute the in an even way to improve the network life time and performance.

\subsubsection{Improve Network Performance}

Standard RPL network performance is analyzed with metrics like convergence time, packet delivery ratio, control traffic overhead, power consumption and latency. Load Imbalance scenario in RPL affect many of these parameters. Hence, load balancing schemes need to provide efficient network performance.

\section{IbRPL: Load Balancing Metric based RPL}

In this section, we present the design and properties of load balancing metric based RPL (lbRPL). The proposal has two steps: Formulation of Load Balancing Index (LBI) and load balanced parent selection for load balanced routing.

\subsection{Load Balancing Index (LBI)}

To quantify load balancing in RPL, we introduce a new routing metric for RPL called Load Balancing Index (LBI). LBI has three steps: setting up network using ETX, measuring parent count $(\mathrm{Pc})$ and calculating remaining energy of the parent $(\mathrm{Pe})$. A leaf node measure how load balanced a parent is in terms of ETX, Pc and Pe. These steps are explained below.

\subsubsection{RPL network setup by ETX}

Load balancing in RPL needs to take node and link metrics that enhance load balancing characteristics. In this step, we take Expected Transmission Count (ETX) for network setup. ETX can be defined as the number of transmissions a node expects to make to a destination in order to successfully deliver a packet. The formula for calculating ETX isIt is calculated by the formula in equation (3).

$$
\mathrm{ETX}=1 /(\mathrm{Df} * \mathrm{Dr})
$$

Where Df is the measured probability that a packet is received by the neighbor and Dr is the measured probability that the acknowledgement packet is successfully received. ETX can be configured at root or node using the ocp etx command.

We chose ETX for network setup than hop count for the reason that ETX is an indicator of link quality. If ETX is low then the link quality is good, i.e., meaning data packets will be delivered in minimum number of transmissions. So low ETX value provides network stability and efficient network performance.

\subsubsection{Parent Count Calculation (Pc)}

In RPL based network, the new node joins the DAG by choosing the parent which broadcasts low rank in its DIO message. The lower rank indicates that the parent is closer to the sink. We want to calculate the parent count $(\mathrm{Pc})$ of a preferred parent before selecting the parent. The path which has less parent count $(\mathrm{Pc})$ will be a step closer in making the network balanced. When $\mathrm{Pc}$ is more the new node will eliminate that parent from selecting. Thus using Pc technique, we can bring load balance to RPL. Parent count scenario is explained in figure 1 . The network consists of one sink node (R) and ten sender nodes $(\mathrm{A}, \mathrm{B}, \mathrm{C}, . ., \mathrm{J}) \mathrm{k}$ is the new node that wants to join the DAG. As node $\mathrm{K}$ receives DIO message from preferred parents $\mathrm{E}, \mathrm{G}$ and J. Each of these preferred parents will have different ranks namely $E=768, G=1024$ and $\mathrm{J}=1280$. Now the new node need to calculate the Pc as part of its RANK update. It has to choose the path which suggests less Pc. The parent count can be obtained by the equation (4).

$$
\text { Parent } \operatorname{count}(\mathrm{Pc})=\frac{\text { Parent } \text { Rank- MinRankIncrease }}{\text { MinRankIncrease }}
$$

Applying eq. (4) in the suggested case, new node $\mathrm{K}$ will have $\mathrm{Pc}$ for preferred parents $E=2, G=3$ and $J=4$. So it would choose $E$ as the preferred parent than $\mathrm{G}$ and $\mathrm{J}$. In this way, any new node that wants 
to join the network will select parents with low Pc. As a result, the routes with more number of parent will be avoided and routes with less parents will be selected. Thus load balancing can be achieved.

\subsubsection{Remaining Energy of Parent (Pe)}

The new node is successful in calculating Pc of the preferred parent. Now, there may be a case where more than one preferred parent will have same number of Pc. The leaf node successfully gets the parent count, then it also takes in to account the remaining parent energy (Pe) of the preferred parents. The new nodes choose parents that have high Remaining parent energy (Pe). High remaining parent energy $(\mathrm{Pe})$ metric provides improved network life time for nodes and DAGs.

\subsection{Parent Selection}

The flow chart of the proposed load balancing metric based RPL (lbRPL) is given in figure 3. It gives the message flow of a load balanced parent selection.

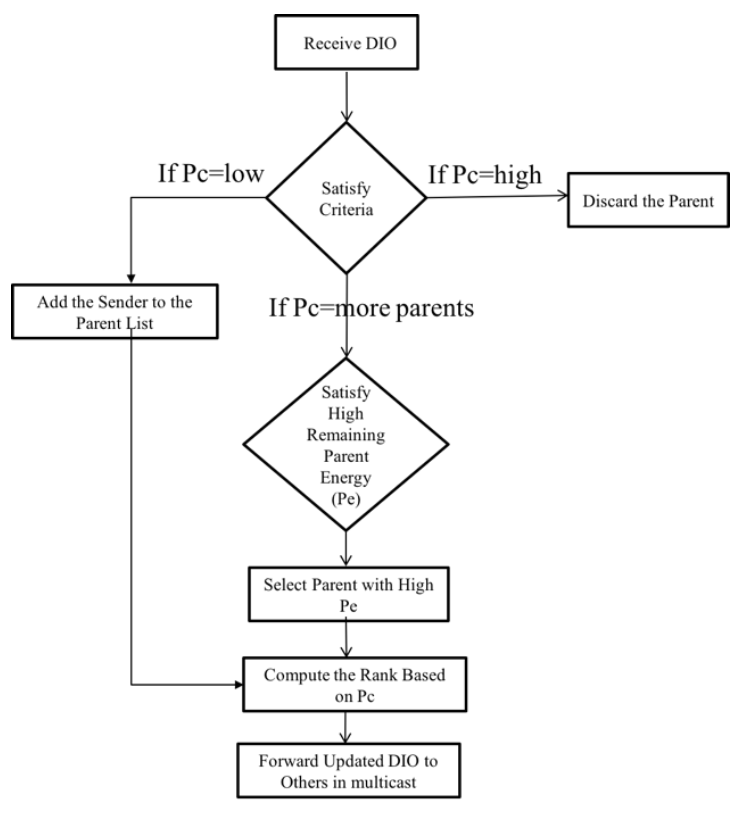

Figure 3: Flow chart for load balanced parent selection

As the new node receives DIO from the parent node, it looks to satisfy the criteria where Parent count $(\mathrm{Pc})$ is low then it updates its rank. If the Pc has the same value for more than one parents, then the condition of high remaining Parent energy $(\mathrm{Pe})$ is satisfied. After satisfying the conditions, the new node selects load balanced parents in hop to routing path.

\section{Simulation Setup and Performance Analysis}

The proposed load balancing metric based routing for RPL (lbRPL) is tested under Contiki OS-COOJA simulator. Contiki is an open source operating system and specifically designed for Internet of Things. It supports IPv6, 6LoWPAN, RPL, etc. It provides the low power communication for highly resource constrained devices. The lossy nature of wireless medium simulated using UDGM is available in COOJA simulator. The simulation parameters are shown in table 1 . The observed results for lbRPL are compared with standard RPL routing metrics such as ETX, Hop Count. The performance results are analyzed for load balancing characteristics such as paren switching, stability, improved life time, packet delivery ratio and control traffic overhead. The values are captured and analyzed from Cooja log view, 6LoWPAN packet Analyzer and collect view methods.
Table 1: Network setup parameters

\begin{tabular}{|l|l|}
\hline \multicolumn{1}{|c|}{ Network Parameters } & \multicolumn{1}{c|}{ Values } \\
\hline Simulation Model & UDGM \\
\hline No. Of Nodes & 100 \\
\hline Area & $200 \mathrm{mx} 120 \mathrm{~m}$ \\
\hline Startup Delay & $65 \mathrm{~s}$ \\
\hline Routing Metrics & ETX, Hop Count and lbRPL \\
\hline Channel & $\begin{array}{l}\text { Channel Check rate } 8 \mathrm{~Hz} \text { and Radio } \\
\text { Channel 26 }\end{array}$ \\
\hline TX and INT Range & Tx $=50 \mathrm{~m}$ and INT $=55 \mathrm{~m}$ \\
\hline Simulation Time & $600000 \mathrm{~ms}$ \\
\hline
\end{tabular}

\subsection{Network stability}

Instability in RPL based networks occur due to frequent parent switching. Frequent parent switching results in low Imin value. Thus frequent network reset generates huge control traffic, energy depletion and poor network performance. The parent switch effects. Here we analyze the rate at which the parent switch happened. It is observed for a number of reconstruction as an indicator. Imin value increases when the network is stable. If Imin holds the highest value means the network is stable and the parent switch reaches minimum level.

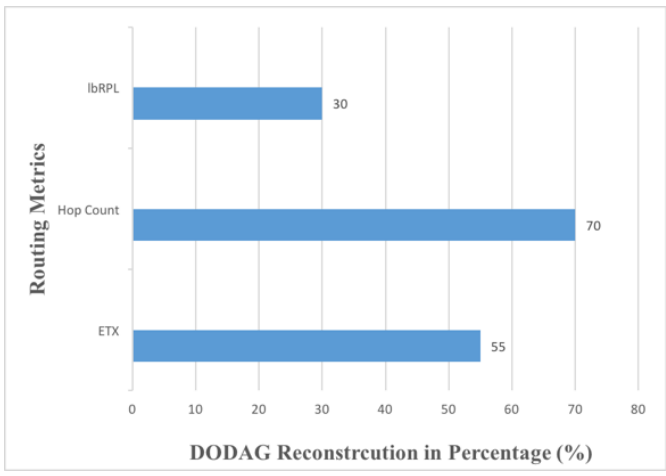

Figure 4: DODAG Reconstruction Percentage

Figure 4, depicts the network stability results in terms of DODAG reconstruction. DODAG reconstruction in our case are due to parent switching. The hop count metric show high (70\%) of parent switching and DODAG reconstruction in comparison to other routing metrics. Our proposed method lbRPL has improved DODAG reconstruction (30\%) and the ETX metric 55\%. Hop count metric takes into account only the minimum hop count, as result more DODAG reconstruction occurs due to link failure as well as load imbalance. ETX metric is more stable than hop count metric due to low link failure. This helps the network to obtain stability after some interval of time. lbRPL takes load balancing metrics so that network becomes stable in short interval time. This stability hol;ds for Imax period. Therefore, lbRPL improved network stability by reducing the number of parent switch.

\subsection{Network Life Time}

Network life time is a prominent criterion for evaluating load balancing for resource constrained devices. The experiment carried out and it is based on number of nodes participating in the network. Figure 5, shows the simulated results. The hop count metric has low node participation (61) and lbRPL metric has high node participation (76). In RPL network, joining of leaving of nodes need to be smooth. Due to load imbalance and related problems, if the leaf nodes face difficulty in parent selection then it results in low node participation. Hop count metric takes only hop count metric which results in low node participation. On the other hand, lbRPL takes in to account, parent count, remaining parent energy and ETX having more node participation. After many intervals, node participation will play a crucial role in extending network life time. When many nodes join the network, schemes to improve network life time such 
as multi parent, multi route, load distribution, etc. can be implemented. Thus improved node participation paves way for improving network life time.

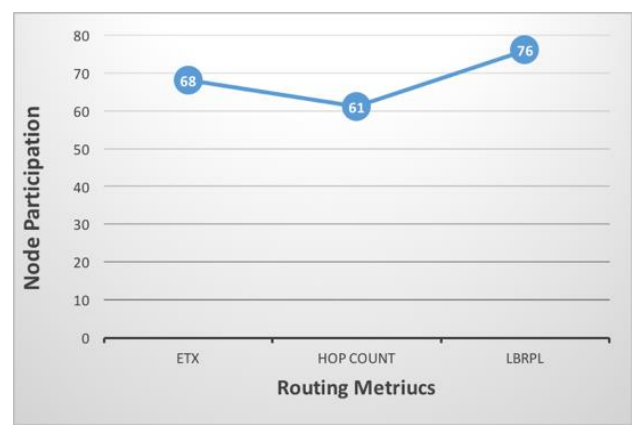

Figure 5: Network Life time through Node Participation

\subsection{Packet Delivery Ratio}

Packet Delivery Ratio observed in the simulation is shown in figure 6 . The obtained results are compared for standard routing metrics ETX and Hop Count and proposed routing metric lbRPL. The PDR for ETX is $96.74 \%$ which is good. On the other hand, PDR for hop count has low value (95\%). lbRPL performs slightly better than hop count and little lower than ETX. The high PDR in routing metrics ETX and lbRPL are due to he fact that these metrics provide link reliability for successful packet delivery than hop count. Our proposed method performs little lower than standard ETX but it also provides network stability by choosing load balanced parents.

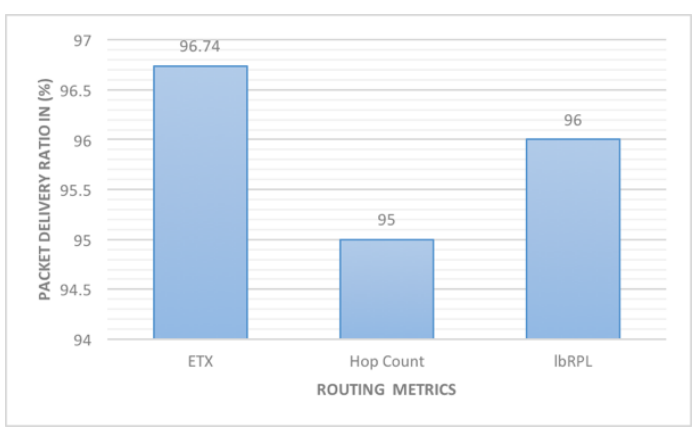

Figure 6: Packet Delivery Ratio

\subsection{Control Traffic Overhead}

Control Traffic overhead is an important parameter to analyze the traffic pattern and the network load. RPL used Internet Control Message Protocol (ICMPv6) to construct and maintain RPL network. Routing decisions are handled by RPL and control messages are handled by ICMPv6. ICMPv6 consist of five messages: DODAG Information Object (DIO), DODAG Information Solicitation (DIS), Destination Advertisement Object (DAO), DAO Acknowledgment (DAO-ACK) and Consistency Check (CC). Load imbalance problems create huge control traffic overhead. The simulated results related to Control Traffic Overhead is shown in Figure 7. It is observed that DIO messages form the major part of the control traffic. Then DIS and DAO and other control traffics are a few. The results suggest high control traffic for ETX and strangely low control traffic for hop count metric. The ETX calculation takes more packet transmission from sender nodes to the sink and viceversa. However, after a few intervals, when the network setup is over, the control message for ETX is few but hop count metric continue to generate. These extremes are well managed by lbRPL. Due to load balancing, the lbRPL shows improved and stable performance during network setup as well after many intervals. Hence, control traffic over head is directly proportional to the stability and load balance of the RPL network.

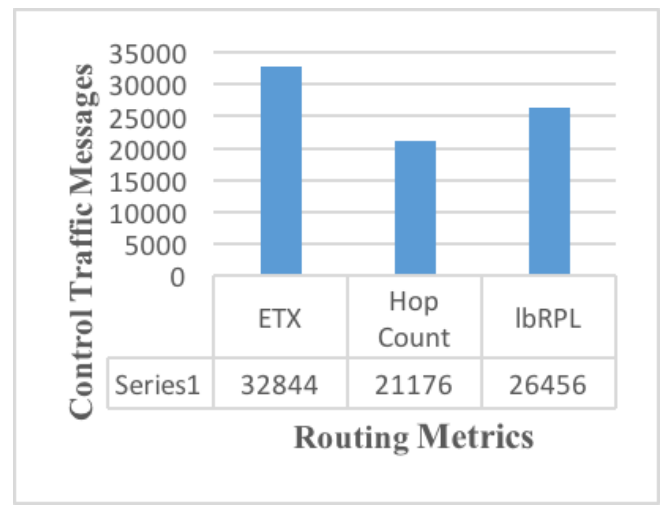

Figure 7: Control Traffic Overhead

\section{Conclusion and Future Work}

Routing in RPL based IoT networks has many schemes for routing optimization, energy efficiency, improve stability and less schemes to tackle load imbalance. In this article, we designed a new metric called Load Balancing Index (LBI), consisting of composite routing metrics ETX, parent count and parent remaining energy. This metric helps the leaf node to select load balanced parents and paths from leaf node to the sink node. The proposed scheme takes in to account node and link metrics that help load balanced routing. The proposed scheme shows improved results. In future, LBI will be extended up to DODAG and Multi DODAG level. In RPL network, every node sends sensed data with different periodic intervals. As a consequence, the radio duty cycle of parent or forwarding nodes are active all the time which results in energy hole or load imbalance. Therefore, new tree structure to group nodes based on reporting time interval or event rate will extend network life time.

\section{References}

[1] D. Miorandi, S. Sicari, F. De Pellegrini, and I. Chlamtac, "Internet of things: Vision, applications and research challenges," $\mathrm{Ad}$ Hoc Networks, vol. 10, no. 7, pp. 1497-1516, 2012.

[2] S. Sivagurunathan et el., Internet of Things for developing smart sustainable cities (SSC): A security Perspective, Connectivity Frameworks for Smart Devices, Computer Communications and Networks (Z. Mahmood (ed), DOI 10.1007/978-3-319-331249_13 307, 2016.

[3] Z. Sheng, S. Yang, Y. Yu, A. Vasilakos, J. McCann, and K. Leung, "A survey on the ietf protocol suite for the internet of things: Standards, challenges, and opportunities," IEEE Wirel. Commun., vol. 20, no. 6, pp. 91-98, 2013

[4] N. Accettura, L. A. Grieco, G. Boggia, and P. Camarda, "Performance analysis of the RPL Routing Protocol," 2011 IEEE Int. Conf. Mechatronics, ICM 2011 - Proc., pp. 767-772, 2011.

[5] B. G. Mamoun Qasem, Ahmed Al-Dubai, Imed Romdhani, "Load Balancing Objective Function in RPL", ROLL - WG INTERNET DRAFT, pp. 1-10, 2017.

[6] R. Jadhav, "Optimization of Parent node selection RPL based Natworks”, ROLL-WG INTERNET DRAFT, pp. 1-11, 2017.

[7] M. R. Palattella et al., "Standardized protocol stack for the internet of (important) things," IEEE Commun. Surv. Tutorials, vol. 15, no. 3, pp. 1389-1406, 2013.

[8] H.-S. Kim, H. Kim, J. Paek, and S. Bahk, "Load Balancing under Heavy Traffic in RPL Routing Protocol for Low Power and Lossy Networks," IEEE Trans. Mob. Comput., vol. 1233, no. c, pp. 1-1, 2016.

[9] Marwa Mamdough et al, "RPL Load balancing via minimum degree spanning tree", IEEE transaction, 2016

[10] X. Liu, J. Guo, G. Bhatti, P. Orlik, and K. Parsons, "Load Balanced Routing for Low Power and Lossy Networks." 
[11] Quan Le, Thu Ngo-Quynh, Thomas Magedanz et al, "RPL based multipath Routing protocols doe Internet of Things", IEEE Xplore, 2014

[12] Minkeun Ha, Kiwoong Kwon, Daeyoung Kim, Peng-Yong Kong, "Dynamic and Distributed Load Balancing Scheme in Multi- gateway based 6LoWPAN", IEEE International Conference on Green Computing, 2015

[13] O. Iova, F. Theoleyre, and T. Noel, "Using multiparent routing in RPL to increase the stability and the lifetime of the network," $A d$ Hoc Networks, vol. 29, no. February, pp. 45-62, 2015.

[14] D. T. Delaney, L. Xu, and G. M. P. O'Hare, "Spreading the load in a tree type routing structure," Proc. - Int. Conf. Comput. Commun. Networks, ICCCN, 2013.

[15] M. N. Moghadam and H. Taheri, "High throughput load balanced multipath routing in homogeneous wireless sensor networks," 22nd Iran. Conf. Electr. Eng. ICEE 2014, no. Icee, pp. 1516-1521, 2014

[16] C. Paper, S. California, D. Gon, G. U. Federal, and S. California, "ALABAMO : A LoAd BAlancing MOdel for RPL ALABAMO A LoAd BAlancing MOdel for RPL," no. May, 2016.

[17] A. Sebastian and S. Sivagurunathan, "Multi Sink RPL based Internet of Things for Emergency Response in Smart Cities," Inter national Journal of Pure and Applied Mathematics, vol. 118, no. 18, pp. 2875-2881, 2018.

[18] A. Sebastian and S. Sivagurunathan, "Bandwidth Allocation based Load Balancing for RPL (BA-LBRPL)," International Journal of Computer Sciences and Engineering, Vol. 2, pp. 76-80, 2018. 